\title{
Erratum to: The apparently anomalous effects of surfactants on interfacial tension in the IBA/water system near its upper critical solution temperature
}

\author{
Renato Lombardo $^{1}$ - Ilaria Calabrese ${ }^{2}$ - Giulia Gelardi ${ }^{3}$ Maria Liria Turco Liveri ${ }^{2}$. \\ John A. Pojman ${ }^{4} \cdot$ Selene Casella $^{2} \cdot$ Tatiana La Monica $^{2}$
}

Published online: 5 November 2016

(C) Springer-Verlag Berlin Heidelberg 2016

Erratum to: Colloid and Polymer Science

DOI 10.1007/s00396-016-3904-9

The original version of this article, unfortunately, contained an error.

The corresponding author accidently neglected to include two of the students as authors. Given in this article is the complete list of authors.

The online version of the original article can be found under at http://dx. doi.org/10.1007/s00396-016-3904-9.

\footnotetext{
John A. Pojman

john@pojman.com

1 Dipartimento di Scienze e Tecnologie Biologiche, Chimiche e Farmaceutiche (STEBICEF), Viale delle Scienze, Ed. 17, 90128 Palermo, Italy

2 Dipartimento di Fisica e Chimica, Viale delle Scienze Ed. 17, 90128 Palermo, Italy

3 Institute for Building Materials, ETH Zurich, Zurich, Switzerland

4 Department of Chemistry, Louisiana State University, Baton Rouge, LA 70803, USA
} 